\title{
Non-intubated video-assisted thoracoscopic tracheal resection and reconstruction for benign distal tracheal stenosis-a case report and review of literature
}

\author{
Benedict Dharmaraj $^{1}{ }^{\wedge}$, Narasimman Sathiamurthy ${ }^{1}$, Narendran Balasubbiah ${ }^{1}$, Amiruddin Nik Mohamed Kamil ${ }^{2}$ \\ ${ }^{1}$ Thoracic Surgery Unit, Department of Surgery, Hospital Kuala Lumpur, Kuala Lumpur, Malaysia; ${ }^{2}$ Department of Anaesthesiology, Hospital Kuala \\ Lumpur, Kuala Lumpur, Malaysia \\ Correspondence to: Dr. Benedict Dharmaraj. Thoracic Surgery Unit, Department of Surgery, Hospital Kuala Lumpur, Jalan Pahang, 50586, Kuala \\ Lumpur, Malaysia. Email: darwin845@yahoo.com.
}

\begin{abstract}
Distal tracheal resection and reconstruction remains a challenging procedure for experienced thoracic surgeons due to its location in the mediastinum. The traditional right posterolateral thoracotomy approach carries significant morbidity in this surgery. With the introduction of video-assisted thoracoscopic surgery (VATS), the outcome of distal tracheal resection and reconstruction has significantly improved, in terms of morbidity and mortality. Non-intubated VATS (NiVATS) tracheal resection and reconstruction is slowly gaining popularity among thoracic surgeons and this approach has shown to have a better outcome than conventional VATS. A 23-year-old female developed distal tracheal stenosis following multiple episodes of intubation for diabetic ketoacidosis. Bronchoscopy revealed a short segment stenosis, 2 to $3 \mathrm{~cm}$ from carina. She underwent multiple tracheal dilatations, but the stenosis recurred. The patient underwent NiVATS tracheal resection and reconstruction, with no complications. She was discharged home well on post-operative day 5 . Repeat bronchoscopy at $1^{\text {st }}, 3^{\text {rd }}$ and $6^{\text {th }}$ months after surgery showed no evidence of re-stenosis. In conclusion, NiVATS is a feasible alternative option for distal tracheal resection and reconstruction which can be performed safely in the hands of experienced surgeons and anaesthetists. To our best knowledge, this is the first reported NiVATS tracheal resection and reconstruction performed in SouthEast Asia.
\end{abstract}

Keywords: Case report; distal trachea resection; non-intubated video-assisted thoracoscopic surgery (NiVATS); benign tracheal stenosis

Received: 01 May 2021; Accepted: 29 July 2021; Published: 30 December 2021.

doi: 10.21037/asj-21-23

View this article at: https://dx.doi.org/10.21037/asj-21-23

\section{Introduction}

Tracheal resection and reconstruction is a complex surgery and is not undertaken by many thoracic surgeons. Distal tracheal lesions pose an even greater challenge due to its location and proximity to the mediastinal structures. Morbidity following thoracotomy for distal tracheal resection has been reported as high as $12.7 \%(1,2)$. Nonintubated video-assisted thoracoscopic surgery (NiVATS) tracheal resection and reconstruction is recently gaining popularity among thoracic surgeons and has shown to have better outcome in terms of shorter surgery time, shorter hospital stay and has no adverse events that are usually associated with general anaesthesia and intubation (2-8). We present a case of a 23 -year-old female with postintubation distal tracheal stenosis, who underwent NiVATS tracheal resection and reconstruction. We present the

\footnotetext{
^ ORCID: 0000-0002-6767-8022.
} 


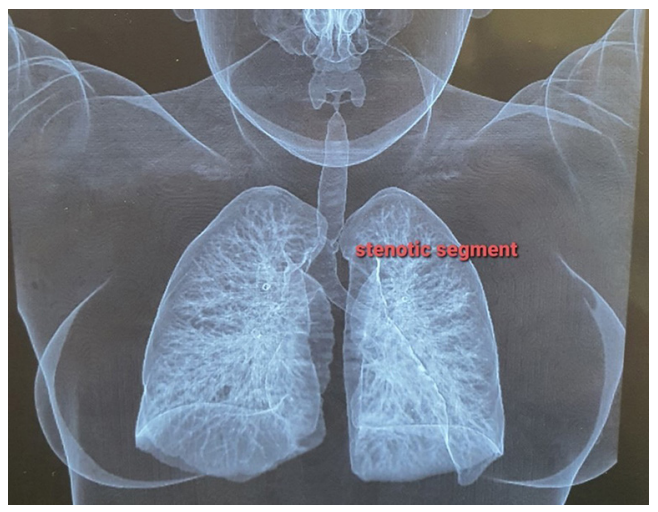

Figure 1 Airway image reconstruction of CECT thorax showing the stenotic segment around $8 \mathrm{~cm}$ from the vocal cords and 2 to $3 \mathrm{~cm}$ from the carina. CECT, contract enhanced computerized tomography.

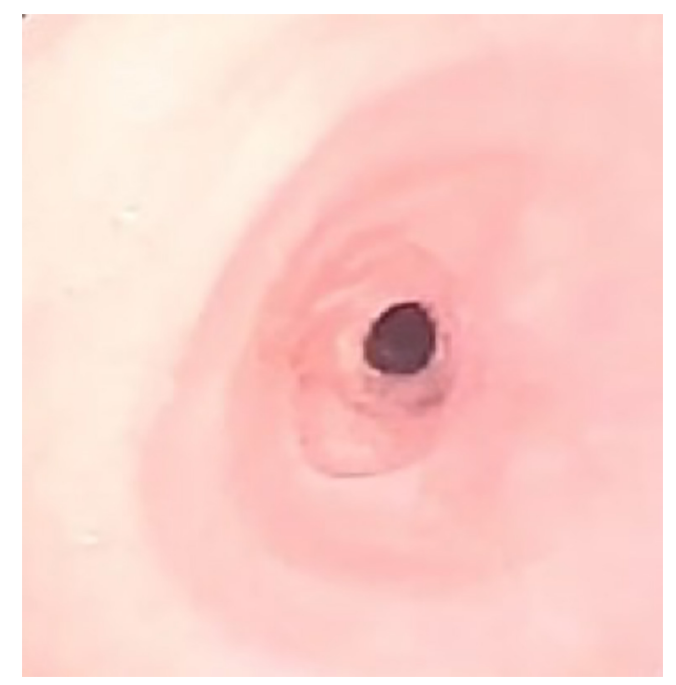

Figure 2 Bronchoscopy view of the trachea before surgery.

following article in accordance with the CARE reporting checklist (available at https://asj.amegroups.com/article/ view/10.21037/asj-21-23/rc).

\section{Case presentation}

A 23 years-old female with a body mass index (BMI) of $24 \mathrm{~kg} / \mathrm{m}^{2}$ and newly diagnosed type- 1 diabetes mellitus, was admitted to a district hospital for diabetic ketoacidosis. She was admitted for a duration of 21 days, in which she required multiple intubations.

Three months following discharge, she had to be intubated twice for dyspnea and stridor within a period of a month. Chest radiograph was unremarkable. A flexible bronchoscopy revealed tracheal stenosis with $80 \%$ narrowing of the lumen, $8 \mathrm{~cm}$ from vocal cord (Figures 1,2). Balloon dilatation and mitomycin application was performed twice, but the stenosis recurred within a month. A computerized tomography (CT) of the neck and thorax revealed a distal tracheal short segment stenosis and tracheal web, 2 to $3 \mathrm{~cm}$ from carina (Figure 2).

She was then referred to our Thoracic Surgery Unit for surgical intervention. We repeated a flexible bronchoscopy to find a hardened stenosis of $<5 \mathrm{~mm}$ lumen at the distal trachea, around $8 \mathrm{~cm}$ from the vocal cords. We did not attempt to dilate the stenosis worrying it may tear the trachea towards the carina and further complicate her management.

Discussion was held between the anaesthetic and the thoracic surgical team to decide on the best approach for the surgery and alternative options. We hypothesized that the main challenge will be to intubate beyond the stenosis, given the lumen size was $5 \mathrm{~mm}$ and the stenosis was just 2 to $3 \mathrm{~cm}$ proximal to the carina. The plans were laid out:

(I) Since patient can lie flat and breath spontaneously sustaining her $100 \%$ saturation, the first plan was to perform right NiVATS distal trachea resection and reconstruction under spontaneous ventilation via supraglottic airway with total intravenous anaesthesia;

(II) If patient desaturate before tracheal transection, an endotracheal tube will be inserted via the supraglottic airway in lateral position and the tip placed above the stenosis for oxygen support, either spontaneous ventilation or with muscle relaxant;

(III) If patient desaturate after tracheal transection, a cross-field flexo-metallic endotracheal tube will be inserted into the left main bronchus via the $3^{\text {rd }}$ or $4^{\text {th }}$ intercostal space by video-assisted thoracoscopic surgery (VATS) (the entire circuit prepared before the surgery commences);

(IV) If all fails, a right thoracotomy will be performed to control the airway by cross-field ventilation.

With these contingency measures discussed, the surgery was commenced.

\section{Anaesthesia process}

A right-sided paravertebral block was planned prior to surgery. The block was performed under ultrasound guidance and $20 \mathrm{~mL}$ of Ropivacaine $0.375 \%$ was given 

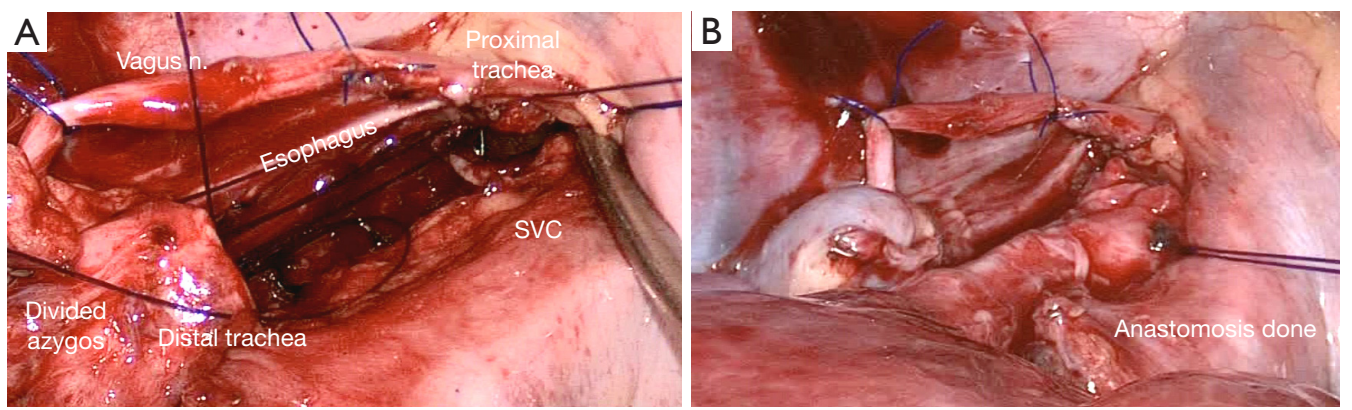

Figure 3 Intraoperative view of the VATS distal trachea resection. (A) The anatomy; (B) the completed tracheal anastomosis using PDS $3 / 0$ suture. SVC, superior vena cava; VATS, video-assisted thoracoscopic surgery.

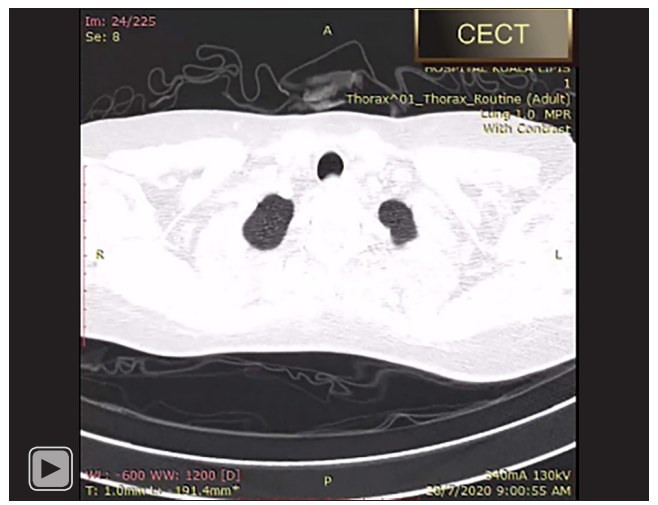

Video 1 Non-intubated video-assisted thoracoscopic surgery (VATS) tracheal resection and reconstruction for distal tracheal resection.

at T2/T3 level. Target controlled infusion (TCI) of Remifentanil and Ketofol (mixture of Ketamine $0.1 \%$ and Propofol $0.9 \%$ ) was used as induction agent. Following induction of anaesthesia, a supraglottic airway (i-gel intersurgical) size 4 was inserted and connected to the anaesthesia breathing circuit. During surgery, anaesthesia was maintained with TCI Remifentanil (Minto model) at $0.7-0.8 \mathrm{ng} / \mathrm{mL}$, TCI Ketofol (Schnider model) at $2-3 \mathrm{mg} / \mathrm{mL}$ and IV Precedex at $0.4 \mu \mathrm{g} / \mathrm{kg} /$ hour. Throughout surgery, spontaneous respiration was maintained, and oxygen $\left(\mathrm{FiO}_{2} 50 \%\right)$ supplied at the flow of $3 \mathrm{~L} / \mathrm{min}$. Depth of anaesthesia was measured with electroencephalogram (EEG) bispectral (BIS) index and maintained between 40-60 by titration of intravenous anaesthesia agents.

\section{Surgical process}

Patient was positioned on left lateral decubitus position.
Lignocaine $2 \%, 5 \mathrm{~mL}$ was administered to skin overlying the right $5^{\text {th }}$ intercostal space, anterior axillary line. A $4-\mathrm{cm}$ skin incision was made at this point and thoracic cavity was accessed. Vagal block was performed with administration of $1 \mathrm{~mL}$ of lignocaine $2 \%+$ Marcaine $5 \%$. Four-level intrathoracic intercostal block (from $\mathrm{T} 2$ to $\mathrm{T} 5$ ) was also given using the same mixture of local anaesthesia, $0.5 \mathrm{~mL}$ to each intercostal nerve.

At this juncture, we noted that the lung was well collapsed, and mediastinal movement was at a bare minimum. The azygous vein was then dissected and transected with a powered vascular stapler, $35 \mathrm{~mm}$. Pleura anterior to trachea was dissected and the vagus nerve was then tacked to the posterior chest wall using prolene $3 / 0$ sutures. Dissection of trachea was then continued. We encountered dense adhesion between the membranous part of trachea and esophagus at the region of stenosis (Figure $3 A$ ).

On-table flexible bronchoscopy was performed to confirm the site of stenosis. Trachea was then transected, and stenotic segment was removed; this step was done meticulously to avoid injury to esophagus that was adherent to the trachea, which required a second port insertion at the right $7^{\text {th }}$ intercostal space, posterior axillary line. Tight haemostatic measures were applied to prevent flooding of distal tracheal opening (Figure $3 A$ ). Tracheal reconstruction was performed with absorbable polydioxanone $3 / 0$ (Monoplus) suture in a continuous manner (Figure 3B). No muscle flap was used to cover the anastomosis (Video 1). Leak test was performed with sterile saline, and only one chest drain was placed after leak test was done. Following skin closure, Grillo's stitch was applied. On-table bronchoscopy was then performed to ensure the integrity of anastomosis.

Total surgery time was $240 \mathrm{~min}$, with total tracheal reconstruction time of $60 \mathrm{~min}$. Oxygen saturation $\left(\mathrm{SPO}_{2}\right)$ 
during surgery was noted to be between $96-98 \%$. The highest end tidal carbon dioxide $\left(\mathrm{ETCO}_{2)}\right.$ was $53 \mathrm{mmHg}$. Blood loss was minimal, estimated less than $20 \mathrm{~mL}$. Patient's vital signs were stable throughout surgery, and there was no need for inotropes. Plan A worked.

\section{Post-operative progress}

Patient was sent to High Dependency Ward immediately after surgery for close monitoring. She was on face mask 5L. Immediate post-operative blood gas revealed $\mathrm{pH} 7.242$,

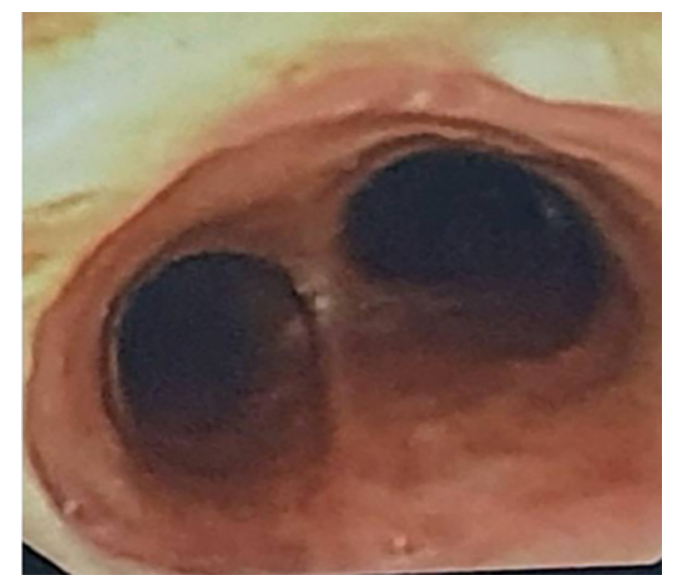

Figure 4 Bronchoscopy view of the trachea post-surgery. partial pressure of carbon dioxide $\left(\mathrm{PCO}_{2}\right) 55 \mathrm{mmHg}, \mathrm{PO}_{2}$ $227 \mathrm{mmHg}$ and blood gas 4 hours later showed $\mathrm{pH}$ 7.362, $\mathrm{PCO}_{2} 45 \mathrm{mmHg}, \mathrm{PO}_{2} 272 \mathrm{mmHg}$. Due to dissection around the esophagus, decision was made to delay her feeding. She was allowed to breathe on nasal prong oxygen at $3 \mathrm{~L} / \mathrm{min}$ around 12 hours after surgery. Post-operative chest radiograph showed both lungs were well expanded.

Patient was transferred to the general ward on postoperative day 1. Nasogastric tube was removed on postoperative day 2, and she was allowed to take clear fluids. IV Paracetamol and patient controlled infusion of morphine was administered for analgesia.

Bronchoscopy was performed on post-operative day 3 (Figure 4), which showed intact anastomosis. Oxygen supplement was then taken off and patient was allowed soft diet. Grillo's stitch was removed, and a soft cervical collar was applied to avoid over-extension of the neck.

Chest drain was taken off on post-operative day 4 and patient was discharged well on post-operative day 5 . A repeat bronchoscopy was done at $1^{\text {st }}, 3^{\text {rd }}$ and $6^{\text {th }}$ month after surgery, and it was noted that the tracheal anastomotic site was intact, with no granulation tissue or evidence of restenosis. Figure 5 depicts timeline of management for the patient.

All procedures performed in this study were in accordance with the ethical standards of the institutional and/or national research committee(s) and with the Helsinki

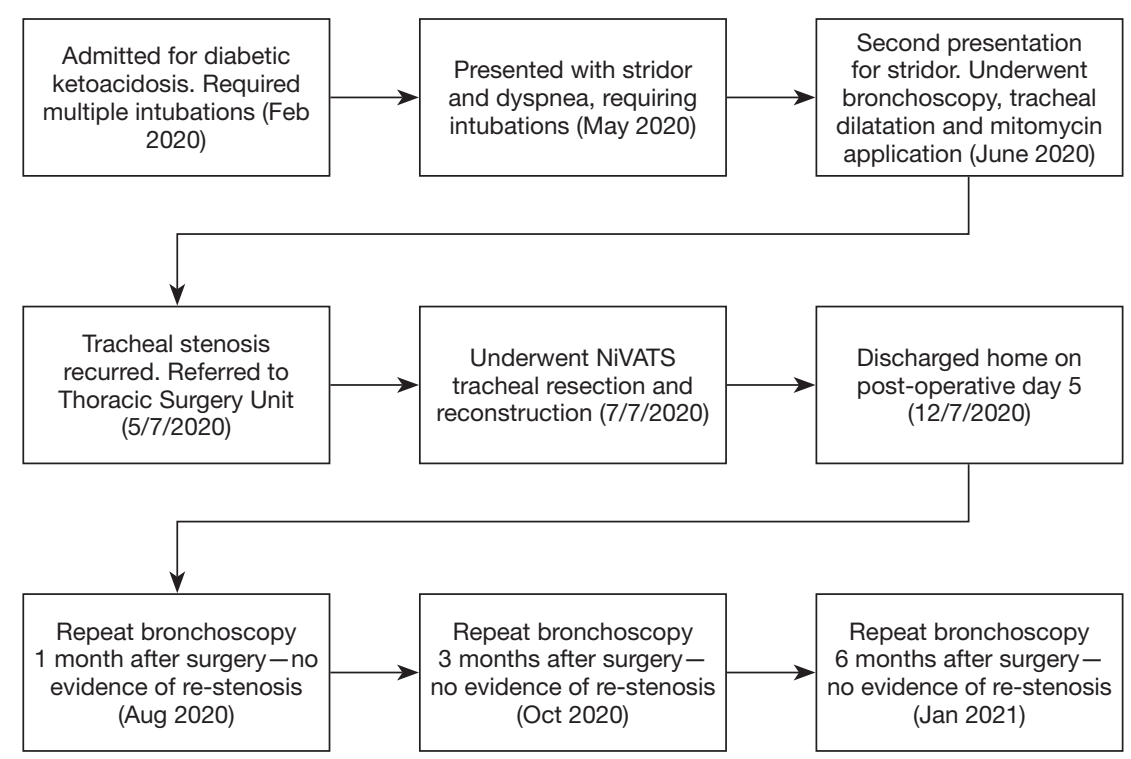

Figure 5 Timeline of historical and current information. NiVATS, Non-intubated VATS. 
Declaration (as revised in 2013). Written informed consent was obtained from the patient for publication of this case report and accompanying images. A copy of the written consent is available for review by the editorial office of this journal.

\section{Discussion}

Tracheal resection and reconstruction is one of the most complex procedures in the field of thoracic surgery and was one of the last procedures to undergo development in this field. Though tracheostomy procedures were well known, tracheal resection and reconstruction were only studied in the 1950s'. Much of the current tracheal reconstruction surgery was developed by Grillo, Cooper, Deverall, Perelman, Dor and Harley (9). Traditionally, median sternotomy or right posterolateral thoracotomy were the choices of approach to distal tracheal surgeries. Both approaches had its limitations, as well as high morbidity and mortality $(1,2,5,7)$. VATS tracheal resection has been proven to be superior to open surgery in terms of less postoperative pain, less morbidity, better preservation of lung function as well as shorter hospital stay $(1,5,7,10)$.

The first VATS circumferential tracheal resection and reconstruction was reported by Nakanishi and Kuruma in 2005 , for a patient with adenoid cystic carcinoma at the mediastinal trachea (11). Few case reports have been published on VATS tracheal resection and reconstruction $(1,2,12)$. The field of thoracic surgery experienced another paradigm shift with the advocation of NiVATS. NiVATS was first reported for diagnostic procedures in 1979 (10). In 1997, Nezu and colleagues reported a series of NiVATS wedge resection in 34 patients (8). To date, Liu et al. reported the largest randomized control trial comparing NiVATS and conventional VATS for various procedure. A total of 354 patients were recruited in this study, with 167 patients undergoing NiVATS. Patients in the NiVATS group had lesser post-operative fasting time, lesser duration for antibiotics, lesser duration of hospital stay, and lesser cytokine release compared to the patients in the conventional VATS group (13). This was clearly reflected with the rate of recovery in our patient after her surgery.

Few papers have reported NiVATS tracheal resection and reconstruction. Huang et al. reported a case of NiVATS tracheal resection and reconstruction for a patient with tracheal glomus tumour. The total surgery time was 130 min, and total reconstruction time was $30 \mathrm{~min}$. There was no documented complication (14). Similarly, Li et al. performed a NiVATS tracheal resection and reconstruction for a patient with tracheal mass. The intraoperative $\mathrm{SPO}_{2}$ was maintained between $97-100 \%, \mathrm{ETCO}_{2}$ was between $40-48 \mathrm{mmHg}$, and estimated blood loss was $30 \mathrm{~mL}$. Total surgery time was $180 \mathrm{~min}$. No post-operative nausea and vomiting was observed in their patient. Patient was allowed to eat 6 hours after surgery and was discharged well on post-operative day 5 (6). Jiang et al. compared the outcome of NiVATS $v s$. conventional VATS for tracheal and carinal reconstruction. Patients in the NiVATS group had shorter operative time, shorter anastomosis time, and shorter hospital stay. Intraoperative $\mathrm{SPO}_{2}$ was comparable in both groups. Though the $\mathrm{ETCO}_{2}$ level was higher in the NiVATS group, no adverse events was seen after surgery (3). Throughout our surgery, we also experienced similar levels of parameters. Our surgery time was longer $(240 \mathrm{~min})$, perhaps due to dense adhesion of the trachea to esophagus caused by previous dilatations and mitomycin application. Furthermore, this was our first NiVATS distal tracheal resection.

Mechanical ventilation is often associated with airway pressure induced injury due to overdistension and will eventually lead to pneumonia $(3,4,6-8)$. Some centres advocate the use of high flow jet ventilation (HFV) during NiVATS. However, with HFV, there is a significant risk of developing acute respiratory distress syndrome $(1,2)$.

Complications due to endotracheal tube or double lumen tube are airway ulceration and injury, sore throat, hoarseness, arytenoid dislocation and rupture $(1,2,8)$. Mortality due to tracheobronchial injury following VATS procedure, due to endotracheal intubation, has been reported to be as high as $22 \%(8)$. For our patient, the tracheal stenosis was hardened with a lumen of $<5 \mathrm{~mm}$ around 2 to $3 \mathrm{~cm}$ from the carina and this made it particularly challenging to preoperatively intubate beyond the stenosis. Decision to perform NiVATS distal tracheal resection naturally became the first option in this case.

General anaesthesia agents and muscle relaxant also contribute to morbidity following VATS. During general anaesthesia, there is cephalic displacement of diaphragm, which in turn causes a decrease in the functional residual capacity (FRC). Due to this, mechanical ventilation with positive-end expiratory pressure is required to maintain ventilation, which can lead to airway pressure induced injury $(2,7,8,13)$. Studies have also shown that there is increase release of inflammatory cytokine in patients who 
underwent conventional VATS $(2,3,6,13)$. This leads to airway oedema. The use of intravenous analgesia also can cause hyperalgesia and vomiting (8). Muscle relaxants are also required in conventional VATS, and this leads to slow recovery of lung function, at times hampering immediate post-operative extubation $(2,3,6,8)$. In context of tracheal resection and reconstruction, it is imperative to extubate the patient immediately after surgery, as prolonged intubation may compromise the tracheal anastomosis. Ventilator related lung injury following VATS has been recorded as high as $4 \%$, with a mortality rate of $25 \%$ (8).

Concerns that may arise among surgeons pertaining to NiVATS tracheal resection and reconstruction will be the management of hypoxia and hypercapnia intra-operatively, as well as exuberant movement of the mediastinum, and coughing during hilar manipulation (7). Surgeons who have embarked on non-intubated tracheal resection and reconstruction advocate the use of laryngeal mask airway (LMA) to maintain oxygenation under spontaneous ventilation $(1,3,6,14)$. In all their cases, patient's $\mathrm{SPO}_{2}$ was maintained between $97-99 \%$ throughout the surgery. And although intraoperative $\mathrm{ETCO}_{2}$ was high (mean 60.1 $\mathrm{mmHg}$ ), the $\mathrm{PCO}_{2}$ at 5 min post-surgery normalized, and no adverse effect was recorded (2). Mediastinal movement and coughing can be minimized by intraoperative vagal block and gentle manipulation of the lung parenchyma to avoid overstretching $(2,6,13)$.

Though NiVATS tracheal resection and reconstruction is a feasible procedure, patient selection is imperative to maximize outcome and minimize complications. Some papers recommend the following criteria for NiVATS: (I) American Society of Anesthesiologists (ASA) 1-2; (II) BMI $<25 \mathrm{~kg} / \mathrm{m}^{2}$; (III) forced expiratory volume at 1 second (FEV1) of more than $60 \%$ of predicted value. Contraindications to NiVATS tracheal resection and reconstruction include bleeding disorders, sleep apnea, cardiac failure, abnormal anatomy, spinal deformity, and pleural adhesions $(3,6,8)$ The other factors that should be taken into consideration in ensuring good outcome in this surgery are the surgeons and the anaesthetist. Surgeons embarking on NiVATS tracheal resection and reconstruction should be experienced, well versed in NiVATS, and have the skill set to perform VATS suturing. The anaesthetist should also be knowledgeable in NiVATS and have the skills to intubate the patient in lateral position should the need arise to convert to general anaesthesia. Good communication between surgeons and anaesthetist is also vital in ensuring a successful surgery with good outcome.

\section{Conclusions}

In conclusion, NiVATS is a feasible alternative option for distal tracheal resection and reconstruction which can be performed safely in the hands of experienced surgeons and anaesthetists. However, it is imperative to carefully select the patients for this surgery. To our best knowledge, this is the first reported NiVATS tracheal resection and reconstruction performed in South-East Asia.

\section{Acknowledgments}

Funding: None.

\section{Footnote}

Reporting Checklist: The authors have completed the CARE reporting checklist. Available at https://asj.amegroups.com/ article/view/10.21037/asj-21-23/rc

Peer Review File: Available at https://asj.amegroups.com/ article/view/10.21037/asj-21-23/prf

Conflicts of Interest: All authors have completed the ICMJE uniform disclosure form (available at https://asj.amegroups. com/article/view/10.21037/asj-21-23/coif). The authors have no conflicts of interest to declare.

Ethical Statement: The authors are accountable for all aspects of the work in ensuring that questions related to the accuracy or integrity of any part of the work are appropriately investigated and resolved. All procedures performed in this study were in accordance with the ethical standards of the institutional and/or national research committee(s) and with the Helsinki Declaration (as revised in 2013). Written informed consent was obtained from the patient for publication of this case report and accompanying images. A copy of the written consent is available for review by the editorial office of this journal.

Open Access Statement: This is an Open Access article distributed in accordance with the Creative Commons Attribution-NonCommercial-NoDerivs 4.0 International License (CC BY-NC-ND 4.0), which permits the noncommercial replication and distribution of the article with the strict proviso that no changes or edits are made and the original work is properly cited (including links to both the formal publication through the relevant DOI and the license). 
See: https://creativecommons.org/licenses/by-nc-nd/4.0/.

\section{References}

1. Li J, Wang W, Jiang L, et al. Video-Assisted Thoracic Surgery Resection and Reconstruction of Carina and Trachea for Malignant or Benign Disease in 12 Patients: Three Centers' Experience in China. Ann Thorac Surg 2016;102:295-303.

2. He J, Wang W, Li J, et al. Video-assisted thoracoscopic surgery tracheal resection and carinal reconstruction for tracheal adenoid cystic carcinoma. J Thorac Dis 2016;8:198-203.

3. Jiang L, Liu J, Gonzalez-Rivas D, et al. Thoracoscopic surgery for tracheal and carinal resection and reconstruction under spontaneous ventilation. J Thorac Cardiovasc Surg 2018;155:2746-54.

4. Li H, Huang D, Qiao K, et al. Feasibility of non-intubated anesthesia and regional block for thoracoscopic surgery under spontaneous respiration: a prospective cohort study. Braz J Med Biol Res 2019;53:e8645.

5. Imanishi N, Tanaka F. Thoracoscopic tracheal resection and reconstruction: video-assisted thoracoscopic surgery as a "tool" toward minimally invasive surgery. J Thorac Dis 2017;9:2895-7.

6. Li S, Liu J, He J, et al. Video-assisted transthoracic surgery resection of a tracheal mass and reconstruction of trachea under non-intubated anesthesia with spontaneous

doi: $10.21037 /$ asj-21-23

Cite this article as: Dharmaraj B, Sathiamurthy N, Balasubbiah N, Kamil ANM. Non-intubated video-assisted thoracoscopic tracheal resection and reconstruction for benign distal tracheal stenosis-a case report and review of literature. AME Surg J 2021;1:30. breathing. J Thorac Dis 2016;8:575-85.

7. Okuda K, Moriyama S, Haneda H, et al. Recent advances in video-assisted transthoracic tracheal resection followed by reconstruction under non-intubated anesthesia with spontaneous breathing. J Thorac Dis 2017;9:2891-4.

8. Zheng H, Hu XF, Jiang GN, et al. Nonintubated-Awake Anesthesia for Uniportal Video-Assisted Thoracic Surgery Procedures. Thorac Surg Clin 2017;27:399-406.

9. Grillo HC. The history of tracheal surgery. Chest Surg Clin N Am 2003;13:175-89.

10. Bertolaccini L, Rocco G. History and development of minimally invasive surgery: VATS surgery. Shanghai Chest 2019;3:16.

11. Nakanishi K, Kuruma T. Video-assisted thoracic tracheoplasty for adenoid cystic carcinoma of the mediastinal trachea. Surgery 2005;137:250-2.

12. Li S, Liu J, He J, et al. Video-assisted thoracoscopic surgery resection and reconstruction of thoracic trachea in the management of a tracheal neoplasm. J Thorac Dis 2016;8:600-7.

13. Liu J, Cui F, Li S, et al. Nonintubated video-assisted thoracoscopic surgery under epidural anesthesia compared with conventional anesthetic option: a randomized control study. Surg Innov 2015;22:123-30.

14. Huang J, Qiu Y, Chen L, et al. Nonintubated Spontaneous Respiration Anesthesia for Tracheal Glomus Tumor. Ann Thorac Surg 2017;104:e161-3. 